\title{
Quasicircular orbits for spinning binary black holes
}

\author{
Harald P. Pfeiffer and Saul A. Teukolsky \\ Department of Physics, Cornell University, Ithaca, New York 14853 \\ Gregory B. Cook \\ Department of Physics, Wake Forest University, Winston-Salem, North Carolina 27109
}

(Received 22 June 2000; published 19 October 2000)

\begin{abstract}
Using an effective potential method we examine binary black holes where the individual holes carry spin. We trace out sequences of quasi-circular orbits and locate the innermost stable circular orbit (ISCO) as a function of spin. At large separations, the sequences of quasi-circular orbits match well with post-Newtonian expansions, although a clear signature of the simplifying assumption of conformal flatness is seen. The position of the ISCO is found to be strongly dependent on the magnitude of the spin on each black hole. At close separations of the holes, the effective potential method breaks down. In all cases where an ISCO could be determined, we found that an apparent horizon encompassing both holes forms for separations well inside the ISCO. Nevertheless, we argue that the formation of a common horizon is still associated with the breakdown of the effective potential method.
\end{abstract}

PACS number(s): 04.25.Dm, 04.70.- s

\section{INTRODUCTION}

The inspiral and coalescence of binary black hole systems is a prime target for upcoming gravitational wave detectors such as the Laser Interferometric Gravitational Wave Observatory (LIGO). Such systems will be circularized by the emission of gravitational waves, and will evolve through a quasi-equilibrium sequence of circular orbits. At the innermost stable circular orbit (ISCO) we expect a transition to a dynamically plunging orbit. It is anticipated that this transition will impart a characteristic signature on the gravitational waveform. It is therefore important to know the orbital frequency at the ISCO, since the corresponding gravitational wave frequency is predominantly just twice this frequency.

Predicting the waveform in detail from the transition at the ISCO to the final merger requires the full machinery of numerical relativity. These calculations require appropriate initial data. Out of the large space of solutions of the initialvalue equations of general relativity, we need an algorithm to select solutions corresponding to black holes in quasicircular orbits. The effective potential method [1] allows one to construct such solutions and to determine the properties of the ISCO.

The effective potential is based on the fact that minimizing the energy of a system yields an equilibrium solution. This follows from the Hamiltonian equations of motion: If the Hamiltonian $\mathcal{H}$ is minimized with respect to a coordinate $q$ and a momentum $p$, then $\dot{q}=\partial \mathcal{H} / \partial p=0$ and $\dot{p}=$ $-\partial \mathcal{H} / \partial q=0$. The energy of two objects in orbit about each other can be lowered by placing the objects at rest at their center of mass. Therefore minimizing the energy with respect to all coordinates and momenta will not yield a circular orbit. To find circular orbits in Newtonian gravity, one can minimize the energy while holding the angular momentum constant. This procedure works as well for a test mass orbiting a Schwarzschild black hole, where one minimizes the Arnowitt-Deser-Misner (ADM) energy. This can be seen as follows. For geodesic motion, one finds [2]

$$
\frac{1}{2} \dot{r}^{2}+\frac{1}{2}\left(1-\frac{2 M}{r}\right)\left(\frac{\widetilde{L}^{2}}{r^{2}}+1\right)=\frac{1}{2} \widetilde{E}^{2}
$$

Here $M$ is the mass of the black hole, $\widetilde{E}$ is the energy per unit rest mass of the test particle as seen from infinity and $\widetilde{L}$ its orbital angular momentum per unit rest mass. Denote the rest mass of the test particle by $M^{\prime}$. Then the ADM energy is simply $E_{\mathrm{ADM}}=M+\widetilde{E} M^{\prime}$, and minimizing $E_{\mathrm{ADM}}$ is equivalent to minimizing $\widetilde{E}$. Hence minimizing the left hand side of Eq. (1) with respect to $r$ yields the radius of circular orbits as a function of angular momentum. Minimization of Eq. (1) with respect to $\dot{r}$ yields $\dot{r}=0$, which is necessary for a circular orbit. From the minimum one finds the energy of the test particle as a function of angular momentum. Obviously, one needs to keep $M$ and $M^{\prime}$ constant during the minimization, so the prescription to compute circular orbits becomes to minimize $E_{\mathrm{ADM}}$ while keeping the angular momentum and the rest masses constant.

These ideas have been formalized as variational principles for finding equilibria for rotating and binary stars in Newtonian gravity. There is also a similar variational principle for rotating stars in general relativity [3]. Binary systems in general relativity are not strictly in equilibrium because they emit gravitational waves. However, for orbits outside the innermost stable circular orbit, the gravitational radiation reaction time scale is much longer than the orbital period. It is therefore a good approximation to treat the binary as an equilibrium system.

In this paper we apply this minimization principle to rotating binary black hole systems. Let the masses of the holes be $M_{1}$ and $M_{2}$, the spins be $\mathbf{S}_{1}$ and $\mathbf{S}_{2}$, and the total angular momentum of the system be $\mathbf{J}$. We exploit the invariance under rescaling of the mass by using dimensionless quantities $M_{1} / M_{2}, \mathbf{S}_{1} / M_{1}^{2}, \mathbf{S}_{2} / M_{2}^{2}$, and $\mathbf{J} / \mu m$, where $m=M_{1}+M_{2}$ denotes the total mass and $\mu=M_{1} M_{2} / m$ the reduced mass. Then we adopt the following straightforward prescription to locate quasi-circular orbits: Minimize the 
scaled ADM energy $E_{\mathrm{ADM}} / m$ with respect to the separation of the holes, while keeping $M_{1} / M_{2}, \mathbf{S}_{1} / M_{1}^{2}, \mathbf{S}_{2} / M_{2}^{2}$, and $\mathbf{J} / \mu m$ constant.

It is somewhat involved to carry out this simple prescription. The computation of the ADM energy becomes more difficult than for the Schwarzschild example above. More importantly, however, no rigorous definitions exist for the mass or spin of an individual black hole in a spacetime containing two black holes. We will address these issues in Sec. II. Ultimately, we must use numerical methods to generate and search among the solutions. Our numerical approach involves root finding, which is also described in Sec. II.

In Sec. III we present the results of the effective potential method. For the interpretation of these results, we need to search for common apparent horizons in our binary black hole data sets. These results are included in Sec. III, too. We discuss our results and conclusions in Secs. IV and V. The Appendix contains the details of the apparent horizon searches.

\section{IMPLEMENTATION}

In order to minimize the ADM energy while keeping $M_{1} / M_{2}, \mathbf{J} / \mu m, \mathbf{S}_{1} / M_{1}^{2}$ and $\mathbf{S}_{2} / M_{2}^{2}$ constant, we need a method to compute the ADM energy as a function of angular momentum, masses and spins of the holes and separation. As a first step we construct initial data $\left(\gamma_{i j}, K_{i j}\right)$ on a hypersurface as described in $[4,5,1]$. Our particular approach assumes conformal flatness of the 3-metric $\gamma_{i j}$ and maximal embedding of the hypersurface, as well as inversion symmetry conditions on the 3-metric $\gamma_{i j}$ and on the extrinsic curvature $K_{i j}$. The effective potential method is independent of these assumptions and works with all methods that compute initial data. For example, in [6], the effective potential method was used without assuming inversion symmetry. In particular, the assumptions of maximal embedding and conformal flatness are not essential but merely convenient-maximal embedding decouples the Hamiltonian and momentum constraints within the initial-data formalism we use, and conformal flatness allows for an analytic solution of the momentum constraints. One disadvantage of conformal flatness is that Kerr black holes do not admit conformally flat 3-metrics, at least for the simple time slicings we are aware of. In [7] it was shown that the Kerr metric is not conformally flat at second order in the spin parameter $S / M^{2}$. Indeed, in Sec. III A we identify this deviation in our results.

Because we assume that the initial hypersurface is maximal, the momentum and Hamiltonian constraints decouple. We follow the Bowen-York [8] prescription to solve the momentum constraint analytically. Then we need only solve one three-dimensional quasi-linear elliptic differential equation, the Hamiltonian constraint. It is solved on a so-called Cadež grid using a multigrid algorithm [5]. The constructed data sets depend on several input parameters, namely the radii and the positions of the throats of the holes in the flat background space, $a_{i}$ and $\mathbf{C}_{i}, i=1,2$, respectively, and their linear momenta and spins, $\mathbf{P}_{i}$ and $\mathbf{S}_{i}, i=1,2$, respectively. We note that in this initial-data prescription, $\mathbf{P}_{i}$ and $\mathbf{S}_{i}$ represent the physical linear and angular momentum of the black hole if it is isolated. We work in the zero momentum frame, where $\mathbf{P}_{2}=-\mathbf{P}_{1}$, and choose $\mathbf{P}_{i}$ perpendicular to $\mathbf{C}_{2}-\mathbf{C}_{1}$ in order to realize a circular orbit. Then the magnitude $P \equiv P_{1}$ $=P_{2}$ is sufficient to describe the linear momenta. Choosing $a_{1}$ as the fundamental length scale, we are left with the following dimensionless input parameters: the ratio of the throat radii, $\alpha=a_{1} / a_{2}$, the dimensionless background separation $\beta=\left|\mathbf{C}_{1}-\mathbf{C}_{2}\right| / a_{1}$, and the dimensionless linear momentum and spins $P / a_{1}$ and $\mathbf{S}_{i} / a_{1}^{2}, i=1,2$, respectively.

From the initial data we can rigorously compute the ADM energy $E_{\mathrm{ADM}}$, the total angular momentum $\mathbf{J}$ and the proper separation between the apparent horizons of each hole, $l$. The total angular momentum is evaluated as in Ref. [1]:

$$
\mathbf{J} \equiv\left(\mathbf{C}_{1}-\mathbf{O}\right) \times \mathbf{P}_{1}+\left(\mathbf{C}_{2}-\mathbf{O}\right) \times \mathbf{P}_{2}+\mathbf{S}_{1}+\mathbf{S}_{2} .
$$

Here $\mathbf{O}$ represents the point about which the angular momentum is defined; it drops out immediately because $\mathbf{P}_{1}=-\mathbf{P}_{2}$. When orbiting black holes have spin, neither the individual spins of the holes nor their orbital angular momentum $\mathbf{L}$ are rigorously defined. We simply take $\mathbf{L}$ to be defined by

$$
\mathbf{L} \equiv \mathbf{J}-\mathbf{S}_{1}-\mathbf{S}_{2}
$$

with $\mathbf{S}_{1}$ and $\mathbf{S}_{2}$ defining the individual spins.

Finally, we need to define the masses of the individual holes. As in Ref. [1], we define the mass of each hole via the Christoudoulou formula:

$$
\begin{aligned}
M_{i}^{2} & =M_{i r, i}^{2}+\frac{S_{i}^{2}}{4 M_{i r, i}^{2}}, \\
M_{i r, i}^{2} & =\frac{A_{i}}{16 \pi},
\end{aligned}
$$

where $A_{i}$ is the area of the event horizon of the $i$ th hole. Clearly this definition is only rigorous for a stationary spacetime. Moreover, we cannot locate the event horizon from the initial data slice alone. Therefore we must resort to using the apparent horizons areas in Eqs. (4) and (5) instead. Apparent horizons can be determined from initial data and in the present case their positions are known to coincide with the throats of the holes [4]. For a stationary spacetime, apparent horizons and event horizons coincide, and in a general, wellbehaved spacetime, the event horizon must coincide with or lie outside of the apparent horizon. In the latter case we will underestimate the mass of the black hole by using the apparent horizon area. Some of the results of this work indicate that this happens for very small separations of the holes.

With the individual masses we can finally define the effective potential as the non-dimensional binding energy of the system:

$$
\frac{E_{b}}{\mu} \equiv\left(E_{\mathrm{ADM}}-M_{1}-M_{2}\right) / \mu .
$$

Since the mass ratio $M_{1} / M_{2}$ is kept constant during the minimization, minimizing $E_{b} / \mu$ is equivalent to minimizing $E_{\mathrm{ADM}} / m$. 
We construct initial data sets starting from the input parameters $\alpha, \beta, P / a_{1}$ and $\mathbf{S}_{i} / a_{1}^{2}$, and compute the physical parameters $E_{b} / \mu, M_{1} / M_{2}, J / \mu m$ and $\mathbf{S}_{i} / M_{i}^{2}$. In order to construct an initial data set with certain physical parameters we have to choose the input parameters appropriately. This requires nonlinear root finding.

Within our effective potential approach, we will search for minima in the binding energy as a function of the separation of the black holes. Fortunately, it is not necessary to solve for a specific proper separation $l / m$. It is sufficient to keep $\beta$ constant during root finding and thus find a binary black hole configuration with some separation $l / \mathrm{m}$. Our goal is to solve the following set of equations [cf. Eqs. (10a)(10d) of Ref. [1]]:

$$
\begin{aligned}
& \frac{M_{1}}{M_{2}}=\left[\frac{M_{1}}{M_{2}}\right] \\
& \frac{S_{1}}{M_{1}^{2}}=\left[\frac{S_{1}}{M_{1}^{2}}\right] \\
& \frac{S_{2}}{M_{2}^{2}}=\left[\frac{S_{2}}{M_{2}^{2}}\right] \\
& \frac{J}{\mu m}=\left[\frac{J}{\mu m}\right] .
\end{aligned}
$$

The bracketed quantities on the right hand sides of Eqs. (7a)-(7c) denote the physical values to be reached, and the expressions on the left-hand side represent functions of the background parameters $\alpha, P / a_{1}, S_{1} / a_{1}^{2}$ and $S_{2} / a_{1}^{2}$ as well as the fixed $\beta$.

For non-rotating holes, Eqs. (7b) and (7c) are trivially satisfied by $S_{1}=S_{2}=0$. For spinning holes this is no longer the case. Hence, it seems one has to solve the complete set of equations (7a)-(7d). However, in any initial data scheme where the physical spins of the black holes are directly parametrized, Eqs. (7b) and (7c) can be eliminated. First, we note again that if the physical spins are directly parametrized, from Eq. (3) we find that we can replace root finding in $J / \mu m$ by root finding in $L / \mu m$. Thus Eq. (7d) is replaced by

$$
\frac{L}{\mu m}=\left[\frac{L}{\mu m}\right] .
$$

In the zero momentum frame, Eqs. (2) and (3) simplify to

$$
\frac{L}{a_{1}^{2}}=\beta \frac{P}{a_{1}} .
$$

Thus we can rewrite $S_{1}$ as

$$
\frac{S_{1}}{a_{1}^{2}}=\frac{S_{1}}{M_{1}^{2}} \frac{M_{1}}{M_{2}} \frac{M_{1} M_{2}}{L} \beta \frac{P}{a_{1}} .
$$

For a solution of Eqs. (7a)-(7c),(8), the first three terms on the right hand side of Eq. (10) take the values of the desired physical parameters, so we can replace them by these parameters throughout the root finding. A similar result holds for $S_{2}$. We perform only two-dimensional root finding, in $\alpha$ and $P / a_{1}$, and set, in each iteration,

$$
\begin{aligned}
& \frac{S_{1}}{a_{1}^{2}}=\left[\frac{S_{1}}{M_{1}^{2}}\right]\left[\frac{M_{1}}{M_{2}}\right]\left[\frac{L}{\mu m}\right]^{-1} \beta \frac{P}{a_{1}}, \\
& \frac{S_{2}}{a_{1}^{2}}=\left[\frac{S_{2}}{M_{2}^{2}}\right]\left[\frac{M_{1}}{M_{2}}\right]^{-1}\left[\frac{L}{\mu m}\right]^{-1} \beta \frac{P}{a_{1}} .
\end{aligned}
$$

For an important subset of spin configurations, even onedimensional root finding is sufficient as can be seen as follows: Consider equal-sized holes with equal spin magnitudes on both holes. If both spins are parallel to the orbital angular momentum or both spins are antiparallel, there exists a symmetry under exchange of the two holes. Therefore $\alpha$ must be equal to 1 and we are left with one free parameter, $P / a_{1}$. If one spin is parallel to the orbital angular momentum and the other spin is antiparallel, however, this property is lost. One hole is co-rotating with the orbital motion and the other hole is counter-rotating. The choice $\alpha=1$ would result in holes with slightly different masses. We thus need twodimensional root finding in $\alpha$ and $P / a_{1}$ for this case.

Each "function evaluation" for the root finding involves the computation of an initial data set $\left(\gamma_{i j}, K_{i j}\right)$. High resolution solutions take between $30 \mathrm{~min}$ and several hours of CPU time on one RS6000 processor. For maximum efficiency, we first perform root finding with a Newton-Raphson method [9] at low resolution data sets. The numerical values for $M_{1} / M_{2}$ and $J / \mu m$ differ slightly between low resolution and high resolution solutions; therefore we solve at low resolution for adjusted values of $\left[M_{1} / M_{2}\right]$ and $[\mathrm{J} / \mu \mathrm{m}]$. With the input parameters found in the low resolution root finding, a high resolution computation is performed to verify that Eqs. (7a) and (7d) are indeed satisfied at high resolution, and to adjust the offset used in the next low resolution root finding. If necessary, this procedure is repeated. On average each complete root finding takes fewer than two high resolution computations.

Following our prescription, we now minimize the binding energy with respect to separation while keeping $M_{1} / M_{2}$, $L / \mu m$ and $\mathbf{S}_{i} / M_{i}^{2}$ constant. The binding energy of a sequence of solutions with these quantities held constant represents a contour of the effective potential. Our code starts at large separation $\beta$ and reduces $\beta$ until a minimum in $E_{b} / \mu$ is bracketed. Then the minimum is located with Brent's method [9], yielding a quasi-circular orbit for the prescribed values of $J / \mu m, M_{1} / M_{2}$, and $\mathbf{S}_{i} / M_{i}^{2}$. Note that each computation of $E_{b} / \mu$ during the minimization along an effective potential contour requires root finding.

By computing quasi-circular orbits for different $J / \mu \mathrm{m}$, but fixed $M_{1} / M_{2}$ and $\mathbf{S}_{i} / M_{i}^{2}$, a sequence of quasi-circular orbits is obtained. A binary black hole that radiates away energy and angular momentum will follow such a sequence approximately, assuming that the spin on each hole remains 


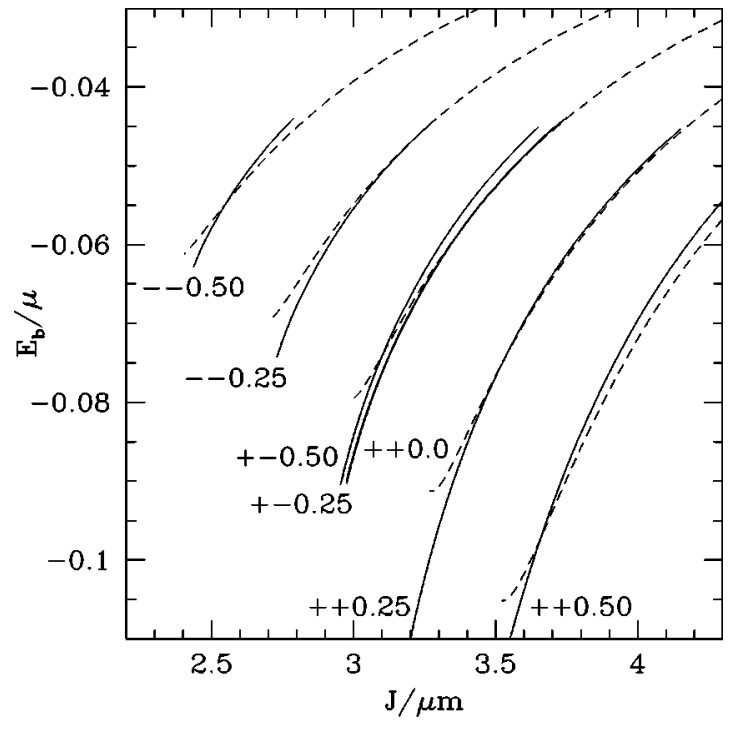

FIG. 1. Sequences of quasi-circular orbits for different spin configurations. Plotted is the binding energy $E_{b} / \mu$ vs the angular momentum $J / \mu m$ along the sequences. The solid lines represent the data; the dashed lines are the results based on (post) ${ }^{2}$-Newtonian theory. As discussed later in this paper, the effective potential method could not locate an ISCO for the ++0.25 and ++0.50 sequences, although we believe each sequence should terminate in one.

constant. We step towards smaller $J / \mu m$, and compute only as many points along each effective potential contour as are required for the minimization. As soon as we do not find a minimum in the effective potential contours anymore we expect to be beyond the innermost stable circular orbit. We trace out some complete effective potential contours around the last value of $J / \mu m$ to check the behavior of these curves.

Finally, from the binding energy $E_{b} / \mu$ and the angular momentum $\mathrm{J} / \mu \mathrm{m}$ along the sequence, we compute the orbital angular frequency as

$$
\Omega=\left.\frac{\partial E_{b}}{\partial J}\right|_{\text {sequence }}
$$

\section{RESULTS}

The parameter space of spinning binary black holes is large - one can vary the mass ratio of the holes as well as spin directions and magnitudes. Astrophysically most interesting are holes that co-rotate with the orbital motion, i.e. with both spins $\mathbf{S}_{i}$ parallel to the orbital angular momentum L. In addition to these co-rotating configurations, we examine configurations with one co-rotating hole and one counterrotating hole, and configurations with two counter-rotating holes. We have the following three families of sequences:

(i) The "++ sequences" with two co-rotating holes.

(ii) The " +- sequences" with one co-rotating and one counter-rotating hole. holes.

(iii) The "-- sequences" with two counter-rotating

We restrict ourselves to equal mass holes, $M_{1}=M_{2}$

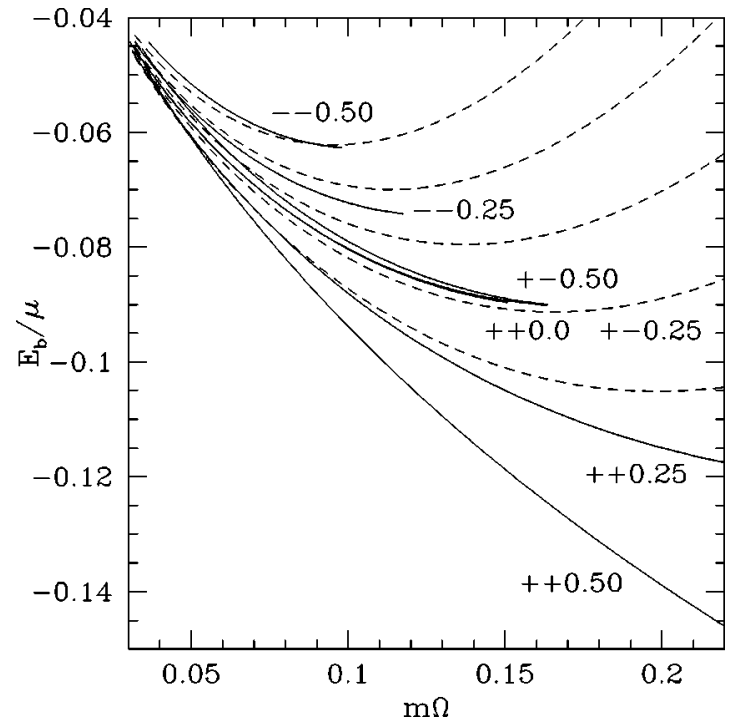

FIG. 2. Sequences of quasi-circular orbits for different spin configurations. Plotted is the binding energy $E_{b} / \mu$ vs the orbital angular frequency $m \Omega$ along the sequences. The solid lines represent the data; the dashed lines are the results based on (post) ${ }^{2}$-Newtonian theory. As discussed later in this paper, the effective potential method could not locate an ISCO for the ++0.25 and ++0.50 sequences, although we believe each sequence should terminate in one.

$\equiv M$, with equal spin magnitudes $S_{1}=S_{2} \equiv S$. As we will see, the assumption of conformal flatness becomes questionable at high spins, so we consider only spin magnitudes $S / M^{2}$ $\leqslant 0.50$. We denote a spin configuration by two plus or minus signs together with a number specifying the spin magnitude on the holes. Thus " ++0.25 " denotes a configuration with two co-rotating holes and spin magnitudes $S_{1} / M^{2}=S_{2} / M^{2}$ $=0.25$.

Quasi-circular orbits were computed for various values of $J / \mu m$ along each sequence. In Fig. 1 the binding energy $E_{b} / \mu$ along each sequence is plotted as a function of the angular momentum $\mathrm{J} / \mu \mathrm{m}$. A binary black hole that loses energy and angular momentum through gravitational radiation moves along such a sequence if the spins of the individual holes remain constant. The dashed lines in Fig. 1 represent the results of (post) ${ }^{2}$-Newtonian theory which we describe in Sec. III A.

Using Eq. (12) we compute the orbital angular frequency. In Figs. 2 and 3, the binding energy and the angular momentum along the sequences are plotted as a function of orbital frequency.

\section{A. Behavior at large separations}

We compare our results to the (post) ${ }^{2}$-Newtonian expansions for spinning holes in quasi-circular orbit that were kindly provided by L. Kidder. The expressions for arbitrary spins and masses are lengthy. If one restricts attention to equal-mass holes, $M_{1}=M_{2}=M, m=2 M, \mu=M / 2$, it turns out that only the sum of the spins enters the (post) $)^{2}$-Newtonian expansions. In terms of 


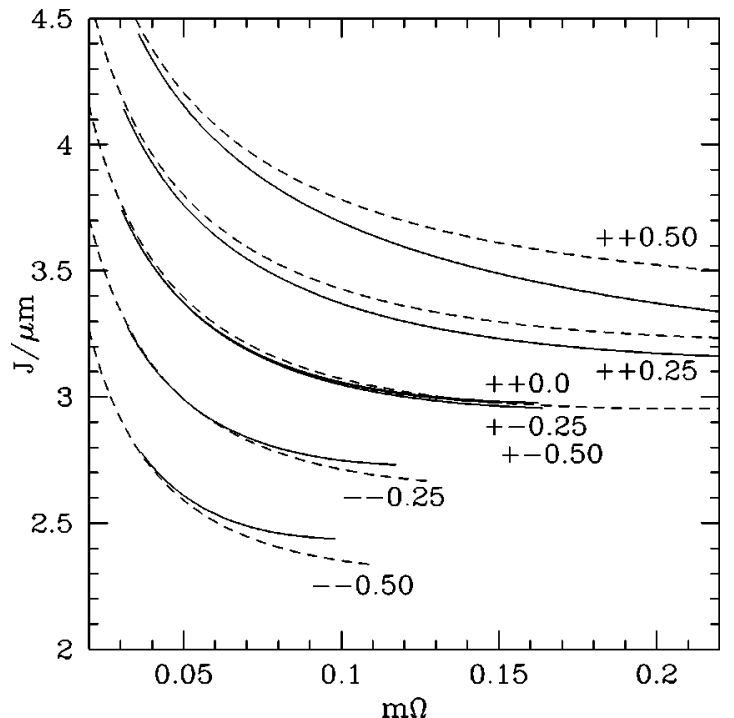

FIG. 3. Sequences of quasi-circular orbits for different spin configurations. Plotted is the angular momentum $\mathrm{J} / \mu \mathrm{m}$ vs the orbital angular frequency $m \Omega$ along the sequences. The solid lines represent the data; the dashed lines are the results based on (post) ${ }^{2}$-Newtonian theory. As discussed later in this paper, the effective potential method could not locate an ISCO for the ++0.25 and ++0.50 sequences, although we believe each sequence should terminate in one.

$$
\mathbf{s} \equiv \frac{\mathbf{S}_{1}+\mathbf{S}_{2}}{M^{2}}
$$

and with $\hat{\mathbf{L}}$ being the unit-vector parallel to $\mathbf{L}$, the (post) ${ }^{2}$-Newtonian expansions become

$$
\begin{aligned}
\frac{E_{b}}{\mu}= & -\frac{1}{2}(m \Omega)^{2 / 3}\left\{1-\frac{37}{48}(m \Omega)^{2 / 3}+\frac{7}{6}(\hat{\mathbf{L}} \cdot \mathbf{s})(m \Omega)\right. \\
& \left.-\left(\frac{1069}{384}+\frac{1}{8}\left[3(\hat{\mathbf{L}} \cdot \mathbf{s})^{2}-\mathbf{s}^{2}\right]\right)(m \Omega)^{4 / 3}\right\}, \\
\left(\frac{J}{\mu m}\right)^{2}= & (m \Omega)^{-2 / 3}\left\{1+2(\hat{\mathbf{L}} \cdot \mathbf{s})(m \Omega)^{1 / 3}\right. \\
& +\left(\frac{37}{12}+\mathbf{s}^{2}\right)(m \Omega)^{2 / 3}+\frac{1}{6}(\hat{\mathbf{L}} \cdot \mathbf{s})(m \Omega) \\
& \left.+\left(\frac{143}{18}-\frac{37}{24}(\hat{\mathbf{L}} \cdot \mathbf{s})^{2}-\frac{7}{8} \mathbf{s}^{2}\right)(m \Omega)^{4 / 3}\right\} .
\end{aligned}
$$

These expressions are plotted in Figs. 1-3 together with our results from the effective potential method. There is remarkable agreement.

The sum $\mathbf{S}_{1}+\mathbf{S}_{2}$ is zero for all +- sequences with equal spin magnitudes, so (post) ${ }^{2}$-Newtonian theory predicts that the +- sequences are identical to the non-rotating sequence. This is remarkable, and indeed, in Figs. 1-3 the +- sequences are close to the ++0.0 sequence. However, a closer look reveals a systematic behavior from which we can gain some insight into our assumptions. For fixed angu-

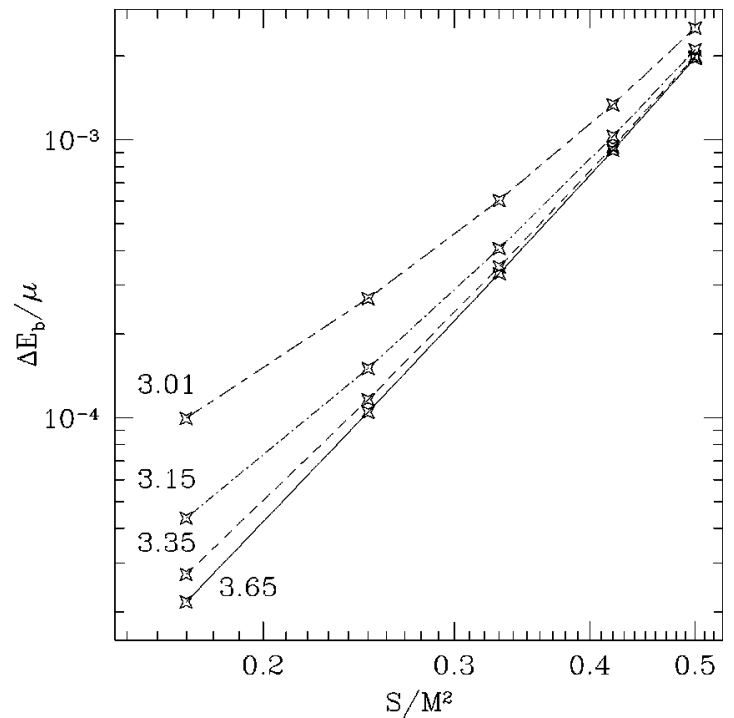

FIG. 4. Difference in binding energy $\Delta E_{b} / \mu$ between +- sequences and non-rotating sequence as a function of spin of the +- sequence for fixed angular momentum $\mathrm{J} / \mu \mathrm{m}$. Each curve is labeled by its value of $J / \mu m$. Here $J / \mu m=3.01$ is very close to the ISCOs that have $J / \mu m \approx 2.98$, and $J / \mu m=3.65,3.35,3.15$ and 3.01 correspond to a separation of $l / m \approx 12.3,9.6,7.7$ and 6.1 , respectively.

lar momentum $\mathrm{J} / \mu \mathrm{m}$, consider the difference in binding energy between a point on a +- sequence and a point on the non-rotating 0.0 sequence:

$$
\Delta E_{b} / \mu(S)=\frac{E_{b}}{\mu}(+-S)-\frac{E_{b}}{\mu}(0) .
$$

In Fig. $4, \Delta E_{b} / \mu(S)$ is plotted as a function of spin for several values of angular momentum $\mathrm{J} / \mu \mathrm{m}$. Here $\Delta E_{b} / \mu$ varies as the fourth power of spin. This might be a physical effect beyond (post) ${ }^{2}$-Newtonian expansions, but for the following reason it seems likely that one of our assumptions introduces a non-physical contribution to $\Delta E_{b} / \mu$, too. Figure 4 strongly suggests that $\Delta E_{b} / \mu$ is converging to a non-zero value as $J / \mu m$ (and thus separation) increases, indicating that there is a contribution to $\Delta E_{b} / \mu$ that is independent of the separation of the holes. For all spin configurations, $E_{b}$ must approach zero in the limit of large separation; therefore any physical contribution to $\Delta E_{b} / \mu$ should decrease with separation. Moreover, a coupling between the holes, physical or unphysical, will give rise to a separation-dependent contribution to $\Delta E_{b} / \mu$. Therefore the separation-independent contribution must be a non-physical effect due to the properties of each isolated hole. A likely candidate is the underlying assumption of conformal flatness. At large separations each hole should resemble a Kerr black hole, which is not conformally flat.

Since the Kerr metric is the unique stationary state for a spinning black hole, if the conformally flat initial data for a single hole were evolved, the metric would relax to the Kerr metric and emit some gravitational radiation. Therefore the total energy contained in our initial data slices is larger than 

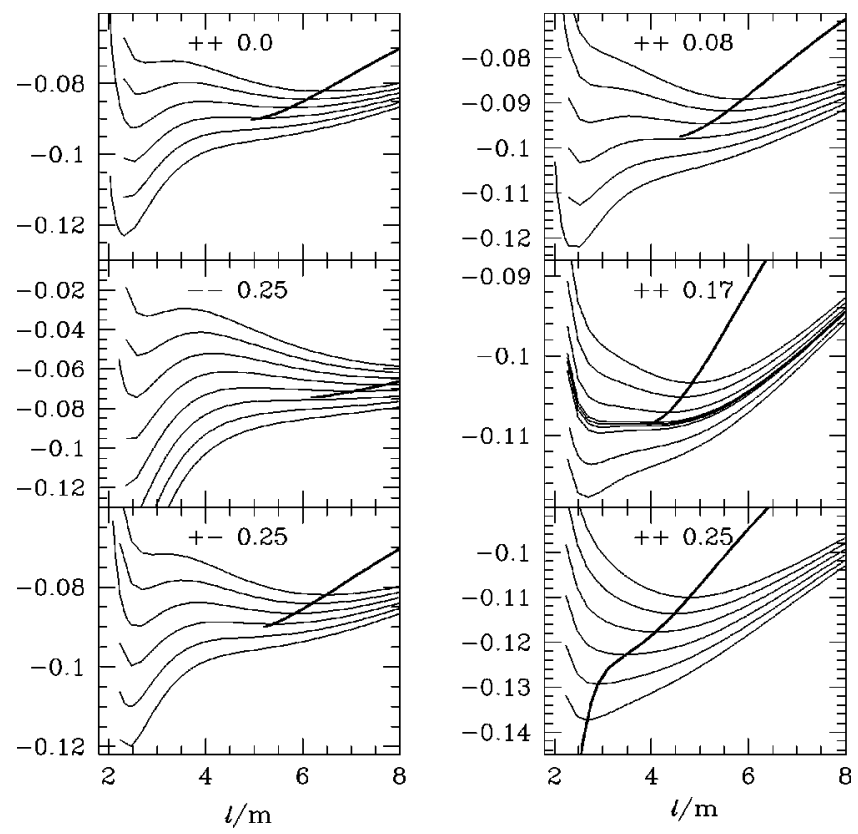

FIG. 5. Constant $J / \mu m$ contours of the effective potential $E_{b} / \mu$ as a function of separation $l / \mathrm{m}$ for various spin configurations. The curves are spaced in steps of $\Delta J / \mu m=0.02$ except for the --0.25 and the ++0.17 configurations, which have steps of 0.04 and 0.01 , respectively. Also plotted is the sequence of quasi-circular orbits connecting the minima of the effective potential.

in a more faithful conformally non-flat data slice and $\Delta E_{b} / \mu$ should be positive, which it indeed is.

We conclude that at large separations $\Delta E_{b} / \mu$ is contaminated by an unphysical contribution because of the conformal flatness assumption. At small separation there might be additional physical contributions beyond the (post) ${ }^{2}$-Newtonian order.

\section{B. Behavior at small separations: ISCO}

In this section we report the key results of this work-the spin dependence of the innermost stable circular orbit. As we will see, the interpretation of our data at small separations is somewhat complicated. At large separations, the assumptions and approximations we have used are reasonable, except for the assumption of conformal flatness when the holes are spinning. At small separations, the interaction between the two black holes becomes relatively strong, and our approximations begin to break down. Near the ISCO, we must evaluate the quality of our assumptions to determine how reliable our results are.

In the neighborhood of each tentative ISCO, we compute a set of complete effective potential contours. These are shown in Fig. 5. In each plot, the binding energy $E_{b} / \mu$ is shown as a function of separation $l / m$ for several different values of angular momentum $\mathrm{J} / \mu \mathrm{m}$. Also plotted is the sequence of quasi-circular orbits passing through the minima of the effective potential. Figure 5 shows the non-rotating sequence ++0.0 , one example each of a -- and a +- sequence, and three ++ sequences with different spin magnitudes.

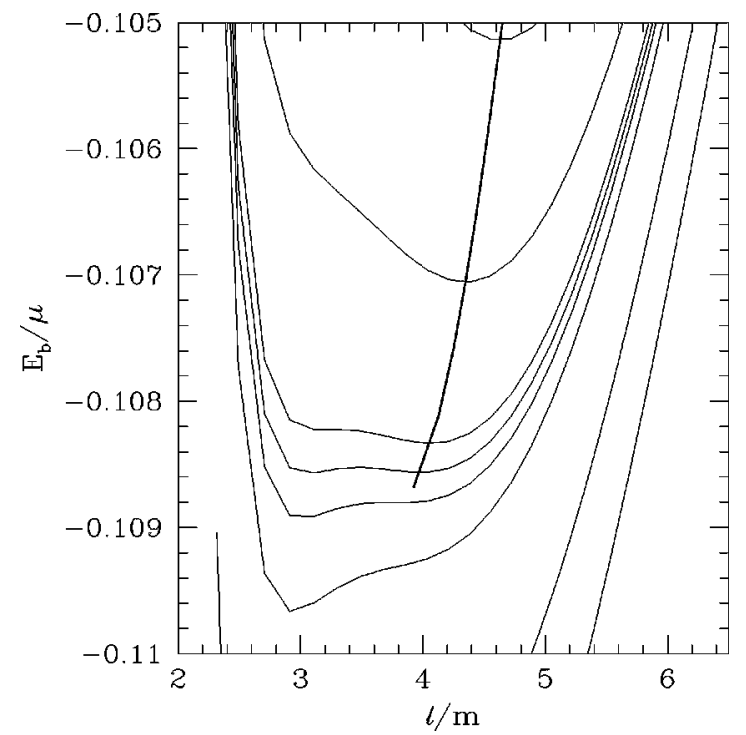

FIG. 6. Enlargement of the ++0.17 sequence of Fig. 5. The displayed effective potential contours (top to bottom) correspond to angular momenta $J / \mu m=3.12,3.11,3.104,3.103,3.102,3.10$, 3.09 and 3.08. Also shown is the sequence of quasi-circular orbits.

Examining the constant $J$ contours of the effective potential for fixed spin configurations, we find that they fall into three regimes separated by critical values that we will label $J_{A}$ and $J_{B}$. Contours with $J>J_{A}$ exhibit a single minimum positioned at large separation $l / m$. This minimum moves inward as the angular momentum decreases; i.e. the holes approach each other as angular momentum and energy are radiated away. We call this the "outer" minimum. As $J$ passes through the critical value $J_{A}$, a new "inner" minimum appears inside the outer minimum. In this region, contours of the effective potential have two minima separated by a local maximum. The maximum corresponds to the well-known unstable circular orbit of a Schwarzschild black hole. As $J$ decreases further, $J_{A}>J>J_{B}$, the maximum moves outward whereas the outer minimum continues to move inward-the quasi-circular orbit associated with the outer minimum continues to shrink. As $J$ passes through the second critical value $J_{B}$ the outer minimum and the maximum meet in an inflection point and disappear. The quasi-circular orbit associated with the outer minimum disappears and this inflection point is identified with the ISCO. For $J<J_{B}$, only the inner minimum remains.

This behavior for the non-rotating sequence was already found in [1]. There, the inner minimum was dismissed as unphysical, since the underlying assumptions become weaker at small separations of the holes and since a common event horizon might form. We will discuss this "unphysical" region and the possibility and consequences of the formation of a common event horizon below. But first we continue discussing the behavior of the effective potential for different spin configurations.

As we increase the spin magnitude for the - - configurations, the two critical angular momentum values $J_{A}$ and $J_{B}$ move away from each other. We see a more pronounced local maximum and the $E_{b}$ curves look similar to the effec- 
TABLE I. Orbital parameters of the innermost stable circular orbit for equal-mass spinning holes. The second through sixth columns give the data obtained in this work; the three columns to the right give the data for a test mass orbiting a Kerr black hole. The results for the ++0.08 and ++0.17 sequences will have larger systematic errors than the other cases (see text).

\begin{tabular}{lcccccccc}
\hline \hline Sequence & $l / m$ & $E_{b} / \mu$ & $m \Omega$ & $J / \mu m$ & $L / \mu m$ & $E_{b} / \mu$ & $L / \mu m$ & $m \Omega$ \\
\hline--0.50 & 7.05 & -0.0628 & 0.100 & 2.438 & 3.438 & -0.04514 & 3.8842 & 0.04935 \\
--0.37 & 6.68 & -0.0687 & 0.107 & 2.595 & 3.335 & -0.04767 & 3.7834 & 0.05319 \\
--0.25 & 6.17 & -0.0743 & 0.120 & 2.730 & 3.230 & -0.05032 & 3.6856 & 0.05727 \\
--0.12 & 5.58 & -0.0815 & 0.139 & 2.865 & 3.105 & -0.05363 & 3.5738 & 0.06242 \\
++0.0 & 4.94 & -0.0901 & 0.166 & 2.976 & 2.976 & -0.05719 & 3.4641 & 0.06804 \\
++0.08 & 4.59 & -0.0975 & 0.186 & 3.042 & 2.882 & -0.05991 & 3.3870 & 0.07237 \\
++0.17 & 3.93 & -0.1087 & 0.235 & 3.103 & 2.763 & -0.06337 & 3.2957 & 0.07793 \\
\hline \hline
\end{tabular}

tive potential of Schwarzschild for a larger interval of angular momenta. The ISCO moves outward to larger separations as spin increases.

Conversely, as we increase the spin magnitude for the ++ configurations the interval $\left(J_{B}, J_{A}\right)$, where two minima and a local maximum exist, becomes smaller. Slightly above $S / M^{2}=0.17, J_{A}$ and $J_{B}$ merge and for $S / M^{2} \geq 0.17$, the regime with two minima and a maximum is not present. Figure 6 illustrates the small interval $\left(J_{B}, J_{A}\right)$ with an enlargement of the ++0.17 sequence. As long as the regime with two minima and a maximum is present, we can still define the ISCO by the inflection point. It moves towards smaller separation of the holes as the spin is increased. However, since the inflection point ceases to exist at some spin magnitude, we cannot define an ISCO for all $S / M^{2}$. Therefore the ++ sequences displayed in Figs. 1-3 do not terminate. Furthermore, we need a more careful analysis to determine whether the ISCO properties for spin magnitudes close to the critical value $S / M^{2} \approx 0.17$ are reliable.

The +- configurations are very similar to the nonrotating one. Given the weak dependence on spin within the +- sequences, this is not surprising. We do not consider the +- configurations further.

Figure 7 and Table I summarize the orbital parameters at the ISCO as a function of spin for the -- sequences and the ++ sequences. The numerical errors in $E_{b} / \mu, L / \mu m$ and $\mathrm{J} / \mu \mathrm{m}$ are less than $1 \%$, while $m \Omega$ and $l / m$ are accurate to a few percent. However, for the ++ sequences the systematic errors of our approach might be much larger. The table also includes ISCO parameters for a test mass orbiting a Kerr black hole obtained from formulas in [10].

\section{Common apparent horizons}

A common event horizon might be responsible for the strange behavior of the effective potential at small separations, because once a common event horizon forms, there are no longer two distinct black holes. It would be helpful to know the critical separation where a common event horizon first forms. However, in order to locate the event horizon, knowledge of the complete spacetime is needed. In the present case, only data on one time slice are available, and so we can only search for common apparent horizons. Since the event horizon must lie outside the apparent horizon, the for- mation of a common apparent horizon places a firm bound on the formation of an event horizon.

Searches for a common apparent horizon were carried out for several spin configurations. Details of the apparent horizon finder and the method used to discern the formation of a common apparent horizon are given in the Appendix. In Table II, the results of the apparent horizon searches are listed.

For fixed spin configurations the common apparent horizon forms at larger separation for larger angular momentum. This can be seen from the --0.25 and ++0.0 sequences. For varying spins and angular momentum close to the ISCO values, the proper separation between the throats at the formation of the common apparent horizon depends weakly on the spin. It decreases from $l / m \approx 2.3$ for the --0.37 sequence down to $l / m \approx 2.0$ for the ++0.17 sequence.

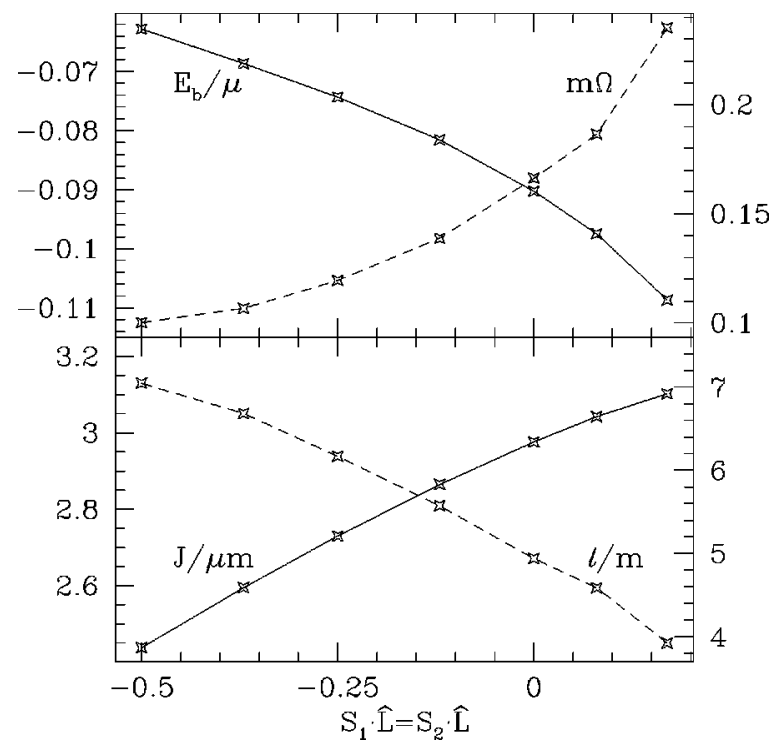

FIG. 7. Values of several physical parameters at the ISCO of the ++ and -- sequences. Plotted are the binding energy $E_{b} / \mu$, the orbital angular frequency $m \Omega$, the total angular momentum $\mathrm{J} / \mu \mathrm{m}$ and the proper separation between the holes, $l / m$, as a function of spin $S / M^{2}$ on the holes. The ++ sequences are plotted along the positive part of the horizontal axis, the -- sequences along the negative part as $-S / M^{2}$. The vertical axes on the left side belong to $E_{b} / \mu$ and $J / \mu m$. 
TABLE II. Summary of the common apparent horizon searches. Listed are the sequences and values of orbital angular momentum for which an apparent horizon search was carried out. The apparent horizon was found to form at a separation $l_{1} / m<l / m<l_{2} / m$.

\begin{tabular}{cccc}
\hline \hline Sequence & $L / \mu m$ & $l_{1} / m$ & $l_{2} / m$ \\
\hline--0.37 & 3.38 & 2.32 & 2.38 \\
--0.25 & 3.10 & 2.20 & 2.25 \\
--0.25 & 3.34 & 2.24 & 2.29 \\
$++0.0^{\mathrm{a}}$ & 0.0 & 1.89 & \\
++0.0 & 2.94 & 2.08 & 2.13 \\
++0.0 & 3.00 & 2.08 & 2.13 \\
++0.08 & 2.84 & 2.03 & 2.08 \\
++0.08 & 2.92 & 2.03 & 2.08 \\
++0.17 & 2.79 & 1.98 & 2.03 \\
++0.25 & 2.70 & 1.96 & 2.01 \\
+-0.25 & 3.00 & & \\
\hline
\end{tabular}

arom [13], which found a critical separation $\beta=4.17$. This corresponds to a proper separation of $l / m \approx 1.89$.

Notice that the segment of parameter space where common apparent horizons form does not include the sequence of quasi-circular orbit configurations. Indeed, the common apparent horizons form at a separation inside the inner minimum where the effective potential increases with decreasing separation.

The search for the onset of common apparent horizons also provides the actual surfaces. In Fig. 8 some apparent horizon surfaces just inside the formation of a common apparent horizon are plotted. The circles represent the throats of the holes. The solid lines represent a cut through the plane of orbital motion of the holes, arrows indicating the direction of linear momentum of the holes. The dashed lines are cuts through the plane perpendicular to the plane of motion and parallel to the spins of the holes. We find that the apparent horizons lag behind the orbital motion, with the amount of lag being larger for counter-rotating than for co-rotating holes.

\section{DISCUSSION}

We found that the effective potential contours at very small separation increase with decreasing separation. This is in contrast to the usual shape of the effective potential for a Schwarzschild or a Kerr black hole, which tends to $-\infty$ at sufficiently small separations.

The common apparent horizon that was found to form at a small separation of the holes might influence the observed effective potential as follows: The event horizon must lie outside the apparent horizon. Therefore a common event horizon must form before a common apparent horizon forms. To accomplish this the event horizons around the individual holes must grow towards this common event horizon. Thus, even before the formation of a common event horizon, the individual event horizons will no longer be close to the individual apparent horizons and the areas of the event horizons of the individual holes must be larger than the areas of their apparent horizons. Therefore, Eqs. (4) and (5) will

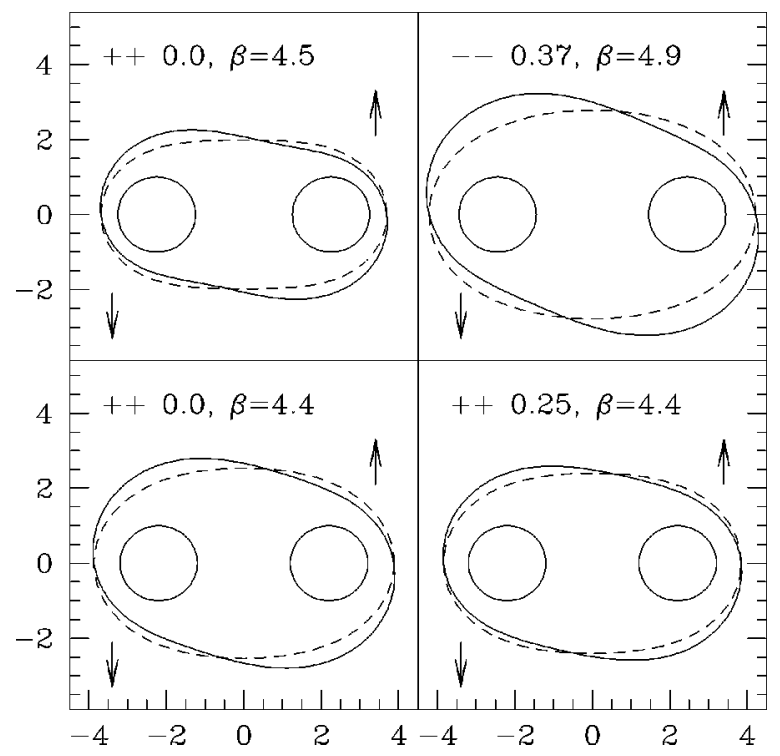

FIG. 8. Shapes of the common apparent horizons for different spin configurations. Circles denote the throats of the holes. The solid lines are cuts in the plane of orbital motion (arrows indicating the direction of motion); the dashed lines represent cuts normal to the plane of motion.

under-estimate the mass of the holes. We denote this underestimate by $\Delta M$. Consider the effect this underestimate of $M$ has on the binding energy. The numerator of Eq. (6) will be over-estimated by a relative amount of

$$
\frac{2 \Delta M}{\left|E_{b}\right|}=\frac{4}{\left|E_{b} / \mu\right|} \frac{\Delta M}{M} \gg \frac{\Delta M}{M} .
$$

At the same time, the denominator of Eq. (6) and the denominator of the scaled angular momentum (7d) change too, leading to an underestimate of the binding energy $E_{b} / \mu$. However, the relative changes of these denominators are only of the order of $\Delta M / M$, so that the overestimate from Eq. (16) dominates. It might well be that this over-estimate is so large that it counter-balances the decreasing effective potential that one might expect in analogy to Schwarzschild or Kerr black holes.

This idea leads to the following picture to explain the observed effective potential curves: At large separation of the holes, the masses of the holes and the effective potential are reliable and we see an effective potential that looks similar to a Schwarzschild black hole. Consider, for example, the ++0.0 sequence: For $J$ slightly above its ISCO value we see the (outer) minimum of the stable quasi-circular orbit and a maximum corresponding to an unstable circular orbit. As $J$ increases, the stable circular orbit moves outwards and the unstable one moves inwards. Once the maximum corresponding to the unstable orbit moves too far in, the $\Delta M / M$ contamination of the effective potential "eats up" the maximum and it disappears.

Now we turn on spin. We found that a common apparent horizon forms at approximately the same proper separation, independent of the spin of the holes. It seems reasonable that the error $\Delta M / M$ is also almost independent of spin. Thus 


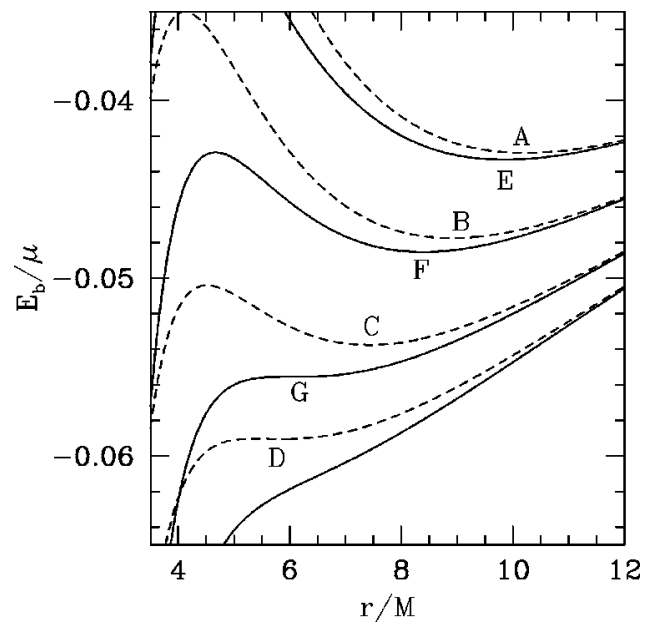

FIG. 9. Illustration of the effects of a systematic underestimation of $E_{b} / \mu$. The dashed lines represent the observed effective potential contours for some values of $J$. The points A, B, and C correspond to circular orbits. The ISCO is at D. Assuming that the true binding energy is smaller, with the deviation increasing as the separation decreases, yields true effective potential contours similar to the solid lines. The true circular orbits are at $\mathrm{E}$ and $\mathrm{F}$ and the true ISCO is at G. We find that the minima of the true contours will lie at smaller separation (for the same $J$ ). The angular frequency is given by $\Omega=d E_{b} / d J$. Using points $\mathrm{A}$ and $\mathrm{B}$, we see that the observed $d E_{b}$ is smaller than the true one, so we under-estimate $m \Omega$. For fixed $J$, true circular orbits will occur at smaller separation, but the true ISCO will appear at larger $J$ than we have observed. These effects counteract each other, making it impossible to predict their effect on the true ISCO.

$\Delta M / M$ becomes significant at roughly the same separation $l / m$ independent of spin. For the - - sequences the ISCO moves to larger separations. Thus the maximum in the effective potential (the unstable orbit) will survive for a larger range of separations and angular momenta $J$. Conversely, for the ++ sequences, the ISCO moves inwards, closer to the separation where $\Delta M / M$ becomes significant. The maximum in $E_{b} / \mu$ is lost almost immediately, and in the extreme limit of $S / M^{2}>0.17$, it does not show up at all.

This scenario is sufficient to capture the complete behavior of the effective potential as a function of $J$ and spin. What does this picture imply for the validity of our ISCO results from Table I? We expect that $\Delta M / M$ decays rapidly with increasing separation, so the ISCO data for the non-rotating sequence ++0.0 as well as for the -- sequences should be sound. However, because $\Delta M$ changes the characteristic behavior for the ++ configurations even for $S / M^{2}<0.17$, the ++ sequences will be affected. Let us consider how these changes affect our estimates of circular orbits.

Figure 9 illustrates the effect of the $\Delta M / M$ contamination on the effective potential contours. As we noted above, the $\Delta M / M$ contamination of the binding energy over-estimates the binding energy of an effective potential contour. Since this error increases as the separation decreases, our estimates for the separation at a given value of angular momentum are also too high, and our estimates of the orbital angular velocity $m \Omega$ are too low. Unfortunately, we cannot determine whether our estimates for the location of the ISCO are too high or too low. While our estimates for the separation of a given orbit are too high, we see that the true ISCO will occur at a larger value of the total angular momentum than we estimate. These effects oppose each other.

The angular momentum at the ISCO, $J / \mu m$, increases with spin for the ++ configurations. It is interesting to examine whether the final black hole resulting from a merger of such a spinning binary black hole can violate the Kerr limit on spin of a black hole. From Eq. (4) we find

$$
M_{i r}^{2}=\frac{M^{2}}{2}\left(1+\sqrt{1-\frac{S^{2}}{M^{4}}}\right) .
$$

By the area theorem, the final irreducible mass must satisfy $M_{i r, f}^{2} \geqslant 2 M_{i r}^{2}$, where equal mass holes were assumed. The final angular momentum cannot exceed the angular momentum at the ISCO, $J_{f} \leqslant J$. With these two constraints and by virtue of the Christoudoulou formula (4), we find

$$
\frac{M_{f}^{2}}{M_{i r, f}^{2}} \leqslant 1+\frac{(J / \mu m)^{2}}{4\left[1+\sqrt{1-\left(S / M^{2}\right)^{2}}\right]^{2}} .
$$

A Kerr black hole has always $M^{2} / M_{i r}^{2} \leqslant 2$ with equality in the extreme Kerr limit. With data from Table I we find for the --0.50 sequence $M_{f}^{2} / M_{i r, f}^{2} \leqslant 1.43$ and for the ++0.17 sequence $M_{f}^{2} / M_{i r, f}^{2} \leqslant 1.61$. These values correspond to spin parameters of $J / M_{f}^{2} \leqslant 0.92$ and $J / M_{f}^{2} \leqslant 0.97$, respectively. Hence the merged black hole might be close to the Kerr limit, but will not violate it.

\section{+- Sequences and conformal flatness}

The (spin $)^{4}$ effect illustrated in Fig. 4 suggests that the assumption of conformal flatness might lead to inaccurate results. This is particularly important for analysis of gravitational waves. As seen in Fig. 4, for spinning holes with $S / M^{2} \sim 0.50$ the assumption of conformal flatness results in an unphysical gravitational wave content of the order of $\sim 2 \times 10^{-3} \mu \sim 5 \times 10^{-4} \mathrm{~m}$. This is less than $0.1 \%$ of the total mass and a few percent of the binding energy $E_{b}$. If the gravitational energy radiated away is less than $1 \%$ of the total mass, then the gravitational wave content due to an unsuitable initial data slice is a significant contamination.

\section{CONCLUSION}

In this work, we have constructed sequences of quasicircular orbits for equal-sized, spinning black holes. At large separations, the results we have obtained match well with (post) ${ }^{2}$-Newtonian expansions, although there is a clear contamination of the data because of the assumption of conformal flatness. The main results of this paper, displayed in Table I and Fig. 7, reveal the behavior of the ISCO for the cases where the spins of the holes are either both co-rotating $(++)$ or counter-rotating $(--)$ with respect to the orbital motion. For co-rotation, the ISCO moves inwards with increasing spin and the orbital angular frequency increases. For 
counter-rotation the ISCO moves outward and the orbital angular frequency decreases. In fact, we find that the orbital angular frequency changes by almost a factor of 2 between the --0.50 sequence and the ++0.08 sequence. We have noted a systematic error in our results that has its origins in an underestimation of the mass of each black hole when they are close together. For the ISCO, this implies that our results are most accurate (ignoring the errors due to conformal flatness) when the holes have large counter-rotating spins, and the error increases as we move to configurations with large co-rotating spins. In fact, the error becomes so large in the ++ sequences that our method cannot locate the ISCO when $S / M^{2} \gtrsim 0.17$.

Our results clearly show the need to give up the simplifying assumption of conformal flatness if we are to construct astrophysically realistic black hole initial data. This is certainly not a new realization, but this is the first time that the effects of the conformal flatness assumption have been seen so clearly in the context of black hole binaries. Work toward more astrophysically realistic initial data has begun [11]. This improvement in the initial data is needed for all separations. It remains to be seen what impact this improvement will have on the process of locating quasi-circular orbits when the holes are close together. It is likely that the systematic underestimate of the mass will still be significant. If so, an improved method for locating quasi-circular orbits and the ISCO will be useful.

\section{ACKNOWLEDGMENTS}

We thank Larry Kidder and Mark Scheel for helpful discussions. This work was supported in part by NSF grants PHY-9800737 and PHY-9900672 and NASA grant NAG57264 to Cornell University and NSF grant PHY-9988581 to Wake Forest University. Computations were performed on the IBM SP2 at Cornell Theory Center and on the Wake Forest University Department of Physics IBM SP2 with support from an IBM SUR grant.

\section{APPENDIX: COMMON APPARENT HORIZONS}

Here we provide details of the apparent horizon $(\mathrm{AH})$ finder. We use the $\mathrm{AH}$ finder described in [12]. The $\mathrm{AH}$ surface is expanded in spherical harmonics up to some order $L$. The apparent horizon, as a marginally outer trapped surface, has everywhere vanishing expansion and is located by minimizing the square of the expansion over the surface. We use convergence with increasing expansion order $L$ to diagnose the formation of a common AH. Therefore high expansion orders $L$ are needed as well as reliable convergence of the minimization routine to the true minimum of the square of the expansion.

The Powell minimization (cf. Ref. [9]) used in [12] is too slow for high-order expansions. We replaced it by a DFP method with finite difference approximations of the Jacobian [9]. For the modest expansion order $L=6$, the DFP method is already 10 times faster than Powell's method.

Furthermore, we take advantage of the symmetries of the AH surface. The holes are located along the $\hat{z}$ axis at

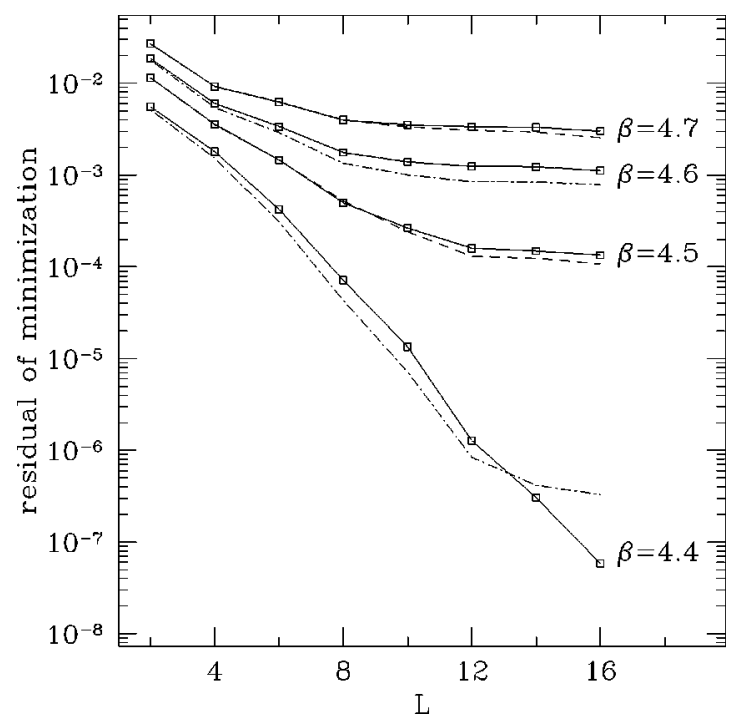

FIG. 10. Residual of the minimization in the $\mathrm{AH}$ finder as a function of expansion order $L$. The number of rays used was $N_{\theta}=64, N_{\phi}=48$. The different solid lines represent different separations of the holes along an effective potential contour with $J / \mu m=3.29$ on the ++0.25 sequence. The dashed lines are the results of minimizations with $N_{\theta}=48, N_{\phi}=32$. The dot-dashed lines show examples of minimizations at lower grid resolution and $N_{\theta}=64, N_{\phi}=48$.

$z= \pm \beta / 2$. Their linear momenta point in the $\pm \hat{x}$ direction and the spins are directed along the $\pm \hat{y}$ axis. It is straightforward to show that these choices imply that the $\mathrm{AH}$ surface is invariant under reflection at the $x z$ plane, $y \rightarrow-y$. This symmetry constrains the coefficients $A_{l m}$ of the expansion in spherical harmonics to be real. Moreover, for the ++ and - - configurations with equal sized holes and equal spin magnitudes, the configuration is symmetric under rotation by $180^{\circ}$ around the $\hat{y}$ axis; this is $(x, y, z) \rightarrow(-x, y,-z)$. Both symmetries together force $A_{l m}=0$ for odd $l$ and $A_{l m}$ to be real for even $l$. Hence the number of free parameters in the minimization routine can be reduced by almost a factor of four.

To prevent convergence to spurious local minima, it is vital that the function that is minimized be as smooth as possible. Therefore we use second order spline interpolation to provide the required data for the $\mathrm{AH}$ finder. Compared to bicubic interpolation, the spline interpolation somewhat decreased the number of iterations needed in the minimization routine, but more importantly it significantly reduced the probability of getting stuck in a local minimum. In addition, many rays were used to reduce the anisotropies introduced by the discrete position of the rays. Finally, we distribute the rays non-uniformly in solid angle. The reason for this is simple: The common AH surface will be very oblate along the $\hat{z}$ axis, since it must encompass the two throats located along the $\hat{z}$ axis. The polar regions of the AH surface are close to the throats and the conformal factor changes rapidly. These regions are particularly important, but the standard distribution uniform in $\cos \theta$ places relatively few rays in the polar regions. Therefore we implemented a procedure that 
distributes the rays in proportion to an arbitrary ray-density function $f(\theta)$. A uniform distribution of rays is represented by $f(\theta)=$ const, whereas we used $f(\theta)=1+\cos ^{2} \theta$, resulting in a doubled density of rays close to the poles.

With the improved AH finder, we performed extensive tests with various numbers of rays. As a rule of thumb, about 10 times more rays as free minimization parameters are necessary to ensure reliable convergence to the true minimum of the square sum of the expansion.

We used expansions up to order $L=16$ and up to 64 $\times 48$ rays (64 in the $\theta$ direction, 48 in $\phi$ ). We perform a set of AH searches, starting at $L=2$ and increasing $L$ by 2 between searches. The result of the previous search is used as the initial guess for the next higher expansion order. Such a set of expansions from $L=2$ to $L=16$ takes typically about $2 \mathrm{~h}$ of CPU time on a RS6000 processor.

A disadvantage of an $\mathrm{AH}$ finder based on a minimization routine is that the minimization routine will always find a minimum. It does not matter whether there actually is a "true" apparent horizon or whether there is only a surface with a small but non-zero expansion. And even for a true $\mathrm{AH}$, the result of the minimization will be non-zero because of the finite grid resolution in the underlying elliptic solver and finite expansion order in spherical harmonics. Therefore we need a method to discern a "true" AH from a mere minimum in the square of the expansion.

For a true $\mathrm{AH}$, the square of the expansion is exactly zero; therefore we expect that the residual of the minimization tends to zero as the resolution of the elliptic solver and the expansion order $L$ are increased. With increasing $L$, the error in the approximation of the surface by spherical harmonics should decrease exponentially. On the other hand, for a mere minimum in the expansion, we expect that the residual of the minimization tends towards a non-zero limit as the resolution of the elliptic solver and the expansion order $L$ is increased. We use this signature to discern the formation of a common apparent horizon.

Figure 10 shows the residual of the minimization for various values of $L$ and different separations $\beta$. The solid lines represent configurations at different separations of the holes. They are labeled by the background separation of the holes, $\beta$. Each solid line represents a set of minimizations with varying expansion order $2 \leqslant L \leqslant 16$ on the same initial data set. At large separations, $\beta \geqslant 4.5$, the residual of the minimization becomes independent of $L$ for large $L$. At small separation, $\beta=4.4$, the residual decreases exponentially through all computed expansion orders up to $L=16-$ a common $\mathrm{AH}$ has formed.

Neither reducing the number of rays nor decreasing the resolution of the Hamiltonian solver changes the convergence behavior significantly. This is illustrated by some examples in Fig. 10. We conclude that for this particular example a common $\mathrm{AH}$ first forms between $\beta=4.4$ and $\beta=4.5$.

Expansions to high order in $L$ are essential for discerning the formation of a common AH. If one had Fig. 10 only up to expansions up to $L=8$, it would be impossible to decide where the common $\mathrm{AH}$ first forms. One would probably conclude that the common $\mathrm{AH}$ forms at larger separations than it actually does.
[1] G. B. Cook, Phys. Rev. D 50, 5025 (1994).

[2] R. M. Wald, General Relativity (University of Chicago Press, Chicago, 1984).

[3] J. B. Hartle and D. H. Sharp, Astrophys. J. 147, 317 (1967).

[4] G. B. Cook, Phys. Rev. D 44, 2983 (1991).

[5] G. B. Cook et al., Phys. Rev. D 47, 1471 (1993).

[6] T. W. Baumarte, Phys. Rev. D 62, 024018 (2000).

[7] A. Garat and R. H. Price, Phys. Rev. D 61, 124011 (2000).

[8] J. M. Bowen and J. York, Jr., Phys. Rev. D 21, 2047 (1980).

[9] W. H. Press, S. A. Teukolsky, W. T. Vetterling, and B. P.
Flannery, Numerical Recipes in $C$ (Cambridge University Press, Cambridge, England, 1992).

[10] J. M. Bardeen, W. H. Press, and S. A. Teukolsky, Astrophys. J. 178, 347 (1972).

[11] R. A. Matzner, M. F. Huq, and D. Shoemaker, Phys. Rev. D 59, 024015 (1999).

[12] T. W. Baumgarte et al., Phys. Rev. D 54, 4849 (1996).

[13] G. B. Cook and A. M. Abrahams, Phys. Rev. D 46, 702 (1992). 